\title{
Cyclophosphamide "metronomic" chemotherapy for palliative treatment of a young patient with advanced epithelial ovarian
}

\section{cancer}

\author{
Riccardo Samaritani1 ${ }^{1}$ Giacomo Corrado*2, Enrico Vizza² and Carlo Sbiroli²
}

\author{
Address: ${ }^{1}$ Division of Medical Oncology, San Carlo IDI Sanità Hospital, Rome, Italy and ${ }^{2}$ Division of Gynaecologic Oncology, National Cancer \\ Institute "Regina Elena", Rome, Italy \\ Email: Riccardo Samaritani - r.samaritani@idi.it; Giacomo Corrado* - g.corrado@ifo.it; Enrico Vizza - e.vizza@ifo.it; \\ Carlo Sbiroli - sbiroli@ifo.it \\ * Corresponding author
}

Published: 15 April 2007

BMC Cancer 2007, 7:65 doi:10.1 186/147/-2407-7-65

This article is available from: http://www.biomedcentral.com//47/-2407/7/65

(C) 2007 Samaritani et al; licensee BioMed Central Ltd.

This is an Open Access article distributed under the terms of the Creative Commons Attribution License (http://creativecommons.org/licenses/by/2.0), which permits unrestricted use, distribution, and reproduction in any medium, provided the original work is properly cited.
Received: 31 July 2006

Accepted: 15 April 2007

\begin{abstract}
Background: Evaluation of the clinical efficacy and tolerance of metronomic chemotherapy as salvage therapy in a young patient with advanced, platinum resistant, ovarian carcinoma and bad performance status.

Case presentation: We tried palliative chemotherapy with daily low dose oral cyclophosphamide with a patient suffering from stage IIIC ovarian cancer that responded to daily cyclophosphamide (CTX) after no response to chemotherapy with paclitaxel and carboplatin as first line and progression after second line with topotecan.

The progression-free survival time on daily low dose oral cyclophosphamide treatment was 65 months without side effects. She was well during the chemotherapy and lived a normal working and social life.

Conclusion: We think that use of low dose of oral CTX should be investigated further as a strategy against tumour progression after standard chemotherapy in patients who are platinum resistant with poor performance status.
\end{abstract}

\section{Background}

Ovarian cancer remains the most common cause of death from a gynaecologic malignancy. In 2005, it is estimated that over 22,000 women will develop ovarian cancer and 16,210 will die as a result [1].

Current treatment of ovarian cancer entails a combination of surgery and chemotherapy. Currently, 1st-line chemotherapy consists of a combination of carboplatin and paclitaxel to which approximately $80 \%$ of women respond [2].
However, despite aggressive surgery and chemotherapy, more than $80 \%$ of patients will relapse and will then be treated with second line chemotherapy with objective responses in about $20 \%$ of patients and even lower percentages of complete responses. This is why the main goal of second and third line chemotherapy is palliative care with the aim to prolong time to progression and to improve quality of life.

Several chemotherapeutic regimens have been used as single agents in phase II trials with patients previously treated 
with cisplatin including paclitaxel, topotecan, liposomal doxorubicin, gemcitabine and oxaliplatin, showing objective response rates ranging from $10-50 \%$ and different toxicity profiles [3].

Many factors play a role when choosing a drug, such as patient compliance, toxicity of previous treatments and treatment costs. Because, at present there are no parameters to predict response to second or third line chemotherapy, the need for less toxic and more efficacious outpatient chemotherapeutic regimens as salvage therapy for advanced ovarian carcinoma is significant.

Recent experimental studies have shown that certain cytotoxic agents, when administered at low and frequent doses are more effective in targeting tumour endothelium than large single bolus doses followed by long rest periods. This is because intra-tumoural vascular endothelial cells, in contrast to the endothelium of quiescent mature blood vessels of normal adult tissues, proliferate rapidly and are vulnerable to cytotoxic agents. However, the long interval between cycles of conventional chemotherapy permits the survival and re-growth of a small number of endothelial cells, allowing tumour angiogenesis to persist. Continuous, low-dose chemotherapy, on the other hand, enhances the anti-angiogenic effect of some cytotoxic agents and may also enhance pro-apoptotic effect in both dividing tumour cells and endothelial cells. A further advantage in the use of continuous low-dose chemotherapy is that it minimises the toxic effects of drugs, allowing more combinations of potentially synergistic selective inhibitors of angiogenesis. The use of dosing regimens that involve the frequent administration of small doses of drugs without extended rest periods has been termed "metronomic" or "high time" chemotherapy [4]. For these reasons we evaluated the clinical efficacy and tolerance of metronomic chemotherapy as salvage therapy in a young patient with advanced ovarian carcinoma platinum resistance and bad performance status.

\section{Case presentation}

In January 2001 a 36 year old woman, nulliparous, menarche at 13 years with a regular cycle came to our Institution because of abdominal pain and increase of abdominal volume. Serum tumour marker tests revealed abnormally high levels of CA-125 (182 IU/ml), but normal levels of CA-19-9 (20,68 IU/ml), CA 15-3 (16 IU/ml) and CEA $(1.1 \mathrm{ng} / \mathrm{ml})$. Ultrasound scan (US) and Computer tomography (CT) of the pelvis was done at our surgical department and revealed a moderate amount of ascites and a conglomeration of the bowel [Fig 1].

Explorative laparotomy revealed one litre of ascites, diffuse peritoneal carcinomatosis and a solid tumour originating from the ovary that formed a mass with extensive adhesions to the uterus, sigmoid colon, and rectum in the pouch of Douglas. There were also numerous metastatic tumours with a diameter of about $3 \mathrm{~cm}$ in the greater omentum and the retroperitoneum. Based on these findings, we decided to only perform biopsy of the tumour. Pathologic examination revealed poorly differentiated serous adenocarcinoma arising from the ovary, FIGO stage IIIC

After surgery, six courses of chemotherapy with paclitaxel $\left(175 \mathrm{mg} / \mathrm{m}^{2}\right)$ and carboplatin (CBDCA: AUC 5) were given every 3 weeks.

At the end of chemotherapy a re-evaluation of the situation by CT scan and serum marker CA $125(102 \mathrm{IU} / \mathrm{ml})$ showed no response to therapy. For this reason we started with a second line of chemotherapy with topotecan $(1,5$ $\mathrm{mg} / \mathrm{m}^{2}$ ) for 5 days every 3 weeks. Unfortunately, fifteen days from the first cycle of chemotherapy the patient underwent surgery again due to an intestinal occlusion.

The second laparotomy showed that the pelvis and the abdomen were completely obstructed by a tumour mass that incorporated the bowel with widespread peritoneal carcinomatosis of diaphragmatic peritonetoneum and on liver surface. This situation with an increase of serum marker Ca 125 (300 IU/ml) showed a clear progression of the disease. Given the extension we decided to perform only a colostomy.

Even if the patient only had one cycle of second line chemotherapy, it was not possible to verify response to further treatment with topotecan, because of the general

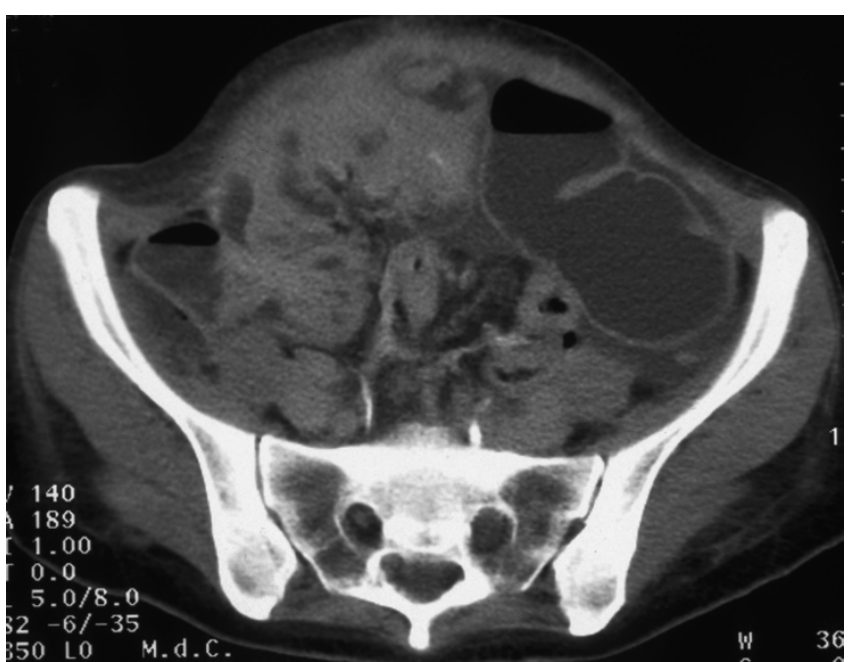

Figure I

Computer tomography (CT) scan of advanced ovarian cancer before treatment showing conglomeration of the bowel. 
deterioration with marked weight loss and anaemia grade 2 (classification by the NCI-CTC).

After thorough discussion with the patient, informed consent was obtained and further salvage chemotherapy was started in August 2001. The regimen of a palliative chemotherapy was oral cyclophosphamide $(50 \mathrm{mg}$ ) every day plus support therapy with epoetin alpha (10000 IU) every day plus vitamins.

Even if evaluation with CT scan and serum marker Ca 125 (90 IU/ml), 3 months after colostomy, showed stabilization of the disease, surprisingly, the patient improved with disappearance of anaemia and weight increase.

For 65 months after the diagnosis of ovarian cancer FIGO stage IIIC platinum resistant the patient continued the chemotherapy with oral cyclophosphamide (50 mg) every day plus vitamins without side effects. She had a stabilization of disease [Fig 2] with a normal blood exams, normal liver and kidney function tests, stabilization of serum marker Ca $125(50 \mathrm{IU} / \mathrm{ml})$ and a good performance status $(E C O G=0)$. In fact she was well and lived a normal working and social life during chemotherapy.

In July 2006 the patient came to our institution because of bladder haemorrhage. We performed a cystoscopy that showed external compression of the bladder with an internal formation of the bladder bottom which bled easily. The biopsy showed a carcinoma of ovarian origin that revealed a progression of disease.

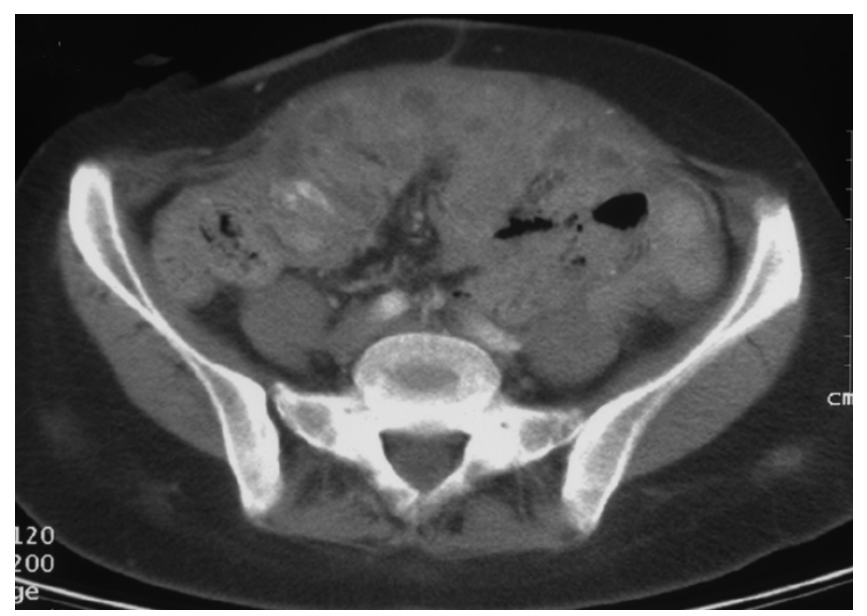

Figure 2

Computer tomography (CT) scan of advanced ovarian cancer after 65 months after diagnosis showing stable disease without bowel occlusion due to successful colostomy.
Unfortunately, the patient died from a severe bladder haemorrhage before trying an other palliative chemotherapy line.

\section{Discussion}

Despite advances in ovarian cancer chemotherapy regimens, there is a high rate recurrence. Second-line ovarian cancer therapy often aims at prolonging survival and time to symptom recurrence. When disease progression or recurrence occurs after primary chemotherapy, choice of subsequent therapy must be individualized.

Due to poor response rates with repeat dosing with platinum agents, second-line treatment for platinum-resistant patients usually involves a cytotoxic agent that uses a different mechanism. These agents include topoisomerase inhibitors (etoposide, topotecan), alkylating agents (ifosfamide, cyclophosphamide), and other chemotherapies (doxorubicin, gemcitabine, docetaxel).

At this time, no one drug combination has been shown to be more efficacious; thus, currently, there is no standardof-care salvage treatment recommendation.

Moreover, these agents used at the maximum tolerated doses may be difficult to administer and are often associated with severe side-effects, sometimes requiring hospitalization.

Not surprisingly, therefore, patient attitudes to toxic chemotherapy regimens for advanced, platinum resistant, ovarian cancer are often negative, as the adverse effects of treatment often seem to outweigh any potential benefits. Thus, the introduction of newer approaches, having improved or at least equivalent efficacy but reduced toxicity, are highly desirable. Therapy must be individualized according to previous response, toxicities, and patient wishes.

Instead of only using short bursts of toxic MTD chemotherapy with long breaks to allow recovery from the harmful side effects, there is now a shift in thinking towards the view that more compressed or accelerated schedules of drug administration using much smaller individual doses than the MTD would be more effective, not only in terms of reducing certain toxicities, but perhaps even improving anti tumour effects as well [5]. The most recent refinement of this concept is called "metronomic" chemotherapy, which refers to the frequent, even daily, administration of chemotherapeutics at doses significantly below the MTD, with no prolonged drug-free breaks.

Metronomic chemotherapy has been defined as a variation of dose-dense therapy with the important exception that it is not necessarily dose intense, the cumulative dose 
might actually be significantly less or equal to MTD-based chemotherapy, thus reducing or perhaps even eliminating in some cases serious drug-induced toxicities, and hence the need for growth-factor support [6]. Unlike MTD chemotherapy that presumably mainly targets (proliferating) tumour cells, frequent or continuous low-dose chemotherapy appears to inhibit preferentially the endothelial cell activity of the tumours' growing vasculature [7]. The basis of this surprising selectivity may have a number of mechanisms.

One is believed to involve inhibition of tumour angiogenesis as a result of chemotherapeutic drug targeting of dividing endothelial cells found in growing tumour-associated blood vessel capillaries.

Moreover, human vascular endothelial cells in vitro are sensitive to the growth inhibiting effects of ultra low concentrations of paclitaxel, in contrast to many other normal cell types or tumour cell types. These effects can be amplified by long-term, continuous exposure, which can also result in apoptosis of endothelial cells [8]. Such effects may be secondary to induction of an endogenous inhibitor of angiogenesis, thrombospondin-1, induced by lowdose chemotherapy by as yet unknown mechanisms, rather than direct inhibition of endothelial cell growth, or survival [9]. In addition, the mobilization, viability and levels of angiogenesis contributing to circulating endothelial progenitor cells may be strongly suppressed, and in a sustained manner, by metronomic chemotherapy [10].

Among the numerous preclinical schedules using different cytotoxic drugs, the oral and daily low-dose administration of cyclophosphamide has been extremely successful in human prostate and breast cancer xenograft mouse models. It has also been successful in spontaneously arising islet cell pancreatic carcinoma, even when the treatment is initiated on advanced, late stage bulky tumours, at least when combined with a targeted anti-angiogenic drug and upfront MTD chemotherapy $[11,12]$. This efficacy is accompanied by absent or low-grade toxicity on tissues otherwise highly sensitive to the respective regimen of the same drug.

For these reasons we used oral daily low-dose cyclophosphamide for our patient, who because of her bad performance status was against undergoing another traditional line of chemotherapy. To our knowledge this is the first report showing the feasibility and the efficacy of cyclophosphamide metronomic chemotherapy for palliative treatment in advanced epithelial ovarian cancer. This is a simple therapy that can be administered on an outpatient basis. Our patient received treatment while performing normal daily activities at home and a high quality of life was maintained.
Probably this good clinical response was due to the antiangiogenetic effect of cyclophosphamide in blocking progression of the disease without any toxicity, together with successful colostomy to improve the general condition of the patient.

The hypothetical anti-angiogenic and anti-tumour effects of low-dose metronomic chemotherapy regimens in mice can be amplified significantly by the concurrent administration of a second drug that is highly specific for activated endothelial cells (e.g., antibodies to vascular endothelial growth factor receptor-2 [VEGFR-2], PEX [C-terminal hemopexin-like domain of matrix metalloproteinase (MMP)-2], anti-VEGF antibodies, and anti-endoglin antibodies). Experiments testing such 'metronomic' schedules of chemotherapy alone or combined with anti-VEGF compounds showed promising anti-tumour activity [13]. A recent phase II study evaluated bevacizumab and low dose 'metronomic' oral cyclophosphamide and found encouraging response rates in 29 patients $(6$ partial response, 17 stable disease, 6 progressive disease) and progression-free survival at 6 months for $47 \%$ of the patients [14]. Despite the encouraging preliminary results reported at the 2005 Meeting of the American Society of Clinical Oncology, this non randomized study presents several limitations: the small number of patients evaluated, the lack of randomization that does not allow discrimination between the activity of 'metronomic' chemotherapy and that of the selective anti-VEGF compound; and, finally, the fact that no correlation with potential markers of angiogenic activity was determined. Currently no definitive clinical data are available because no randomized trial comparing conventional versus metronomic with dose-dense schedules has been concluded [15].

\section{Conclusion}

In conclusion, low dose, oral CTX, demonstrated significant efficacy in this patient with pre-treated advanced ovarian cancer. Theoretically, treatments aimed at inhibiting angiogenesis should be chronically administered for a prolonged period. We think that use of oral CTX should be investigated further, with or without a second drug that is highly specific for activated endothelial cells, as a strategy against tumour progression after standard chemotherapy in patients who are platinum resistant with poor performance status.

\section{Competing interests}

The author(s) declare that they have no competing interests.

\section{Authors' contributions}

SR performed chemotherapy and decide to submit the manuscript for publication. 
CG prepared the manuscript, reviewed the literature, prepared the figure and edited the report.

VE and SC performed surgical treatment.

All authors read and approved the final manuscript.

\section{Acknowledgements}

Written consent was obtained from the patient for publication of study.

\section{References}

I. Sood AK, Abu-Rustum NR, Barakat RR, Bodurka DC, Brown J, Donato ML, Poynor EA, Wolf JK, Gershenson DM: Fifth International Conference on Ovarian Cancer: challenges and opportunities. Gynecol Oncol 2005, 97(3):916-923.

2. Eltabbakh GH: Recent advances in the management of women with ovarian cancer. Minerva Ginecol 2004, 56(I):8I-89.

3. Latorre A, De Lena M, Catino A, Crucitta E, Sambiasi D, Guida M, Misino A, Lorusso V: Epithelial ovarian cancer: second andthird line chemotherapy. Int J Oncol 2002, 2I(I): I79-I86.

4. Gasparini G: Metronomic scheduling: the future of chemotherapy? Lancet Oncol 200I, 2:733-740.

5. Kerbel RS, Klement G, Pritchard KI, Kamen BA: Continuous lowdose anti-angiogenic (metronomic) chemotherapy: from the research laboratory into the oncology clinic. Ann Oncol 2002, 13:12-I5.

6. Kerbel RS, Kamen BA: The anti-angiogenic basis of metronomic chemotherapy. Nat Rev Cancer 2004, 4:423-436.

7. Gately S, Kerbel R: Antiangiogenic scheduling of lower dose cancer chemotherapy. Cancer J 2001, 7:427-436.

8. Bocci G, Nicolaou KC, Kerbel RS: Protracted low-doseeffects on human endothelial cell proliferation and survival in vitroreveal a selective antiangiogenic window for various chemotherapeutic drugs. Cancer Res 2002, 62:6938-6943.

9. Bocci G, Francia G, Man S Lawler J, Kerbel RS: Thrombospondin I, a mediator of the antiangiogenic effects of low-dose metronomic chemotherapy. Proc Natl Acad Sci USA 2003, 100:12917-12922.

10. Bertolini F, Paul S, Mancuso P, Monestiroli S, Gobbi A, Shaked Y, Kerbel RS: Maximum tolerable dose and low- dose metronomicchemotherapy have opposite effects on the mobilization and viability of circulating endothelial progenitor cells. Cancer Res 2003, 63(15):4342-4346.

II. Man S, Bocci G, Francia G, Green SK, Jothy S, Hanahan D, Bohlen P, Hicklin DJ, Bergers G, Kerbel RS: Antitumor effects in mice of low-dose (metronomic) cyclophosphamide administered continuously through the drinking water. Cancer Res 2002, 62(10):273I-2735.

12. Pietras K, Hanahan D: A multitargeted, metronomic, andmaximumtolerated dose 'chemo-switch' regimen is antiangiogenic, producing objective responses and survival benefit in a mouse model of cancer. J Clin Oncol 2005, 23:939-952.

13. Klement G, Baruchel S, Rak J, Man S, Clark K, Hicklin DJ, Bohlen P, Kerbel RS: Continuous low-dose therapy with vinblastine and VEGF receptor-2 antibody induces sustained tumour regression without overt toxicity. J Clin Invest 2000, 105:R I5-R24.

14. Garcia AA, Oza AM, Hirte H, Fleming G, Tsao-Wei D, Roman L, Swenson S, Gandara D, Scudder S, Morgan R: Interim report ofa phase II clinical trial of bevacizumab (Bev) and low dose metronomic oral cyclophosphamide (mCTX) in recurrent ovarian (OC) and primary peritoneal carcinoma: A California Cancer Consortium Trial. J Clin Oncol (Meeting Abstracts) 2005, 23:5000

15. Gasparini G, Longo R, Toi M, Ferrara N: Angiogenic inhibitors: a new therapeutic strategy in oncology. Nat Clin Pract Oncol 2005, 2(I I):562-577.

\section{Pre-publication history}

The pre-publication history for this paper can be accessed here: http://www.biomedcentral.com/1471-2407/7/65/prepub Publish with Bio Med Central and every
scientist can read your work free of charge

"BioMed Central will be the most significant development for disseminating the results of biomedical research in our lifetime. "

Sir Paul Nurse, Cancer Research UK

Your research papers will be:

- available free of charge to the entire biomedical community

- peer reviewed and published immediately upon acceptance

- cited in PubMed and archived on PubMed Central

- yours - you keep the copyright

Submit your manuscript here:

http://www.biomedcentral.com/info/publishing_adv.asp
BioMedcentral 